\title{
Sur quelques groupes de LiE abéliens sur un corps de caractéristique $p>0$
}

(Rectifications)

\author{
Par Jean Dieudonné, Nancy
}

Par suite d'une erreur de calcul, les résultats énoricés dans les $n^{\text {os }} 6$ et 7 de l'article cité dans l' Arch. Math. 5, 274-281 (1954) sont erronés. En fait, il est facile de voir que le groupe $W_{2}^{*}$ est isomorphe au produit direct $W_{1}^{*} \times W_{1}$, l'isomorphie étant définic par $u_{1}\left(x_{1}, x_{2}\right)=x_{1}, u_{2}\left(x_{1}, x_{2}\right)=x_{2} /\left(1+x_{1}^{p}\right)$. Plus généralement, on peut montrer que le groupe $W_{n}^{*}$ est isomorphe au produit $W_{1}^{*} \times W_{n-1}$.

Je signale aussi que dans le $\mathrm{n}^{0} 5$ de l'article, il faut lire $Z_{1}=\Phi\left(Y_{0}, \ldots, Y_{r}, X_{02}, \ldots, X_{r 2}\right)$ à la ligne 13 du bas, p. 278, Il y a aussi un lapsus dans les lignes $1-2$ du haut de la p. 278 , où il faut prendre $t_{i-1}=x_{i}, t_{i-1}^{\prime}=y_{i}$ pour $i \geq 2, t_{0}=1+x_{1}, t_{0}^{\prime}=1+y_{1}$, et remplacer $\varphi_{k}^{*}-1$ par $\varphi_{k}^{*}$ pour $k \geq 1$. Enfin, les considerations des $n^{08} 2$ et 5 relatives aux lois de composition canoniques sont basés sur un raisonnement inexact du mémoire cité [2], et doivent être supprimées.

Eingegangen am 15. 10. 1954 